\title{
Is energy healing an effective non -pharmacological therapy for improving symptom management of chronic illnesses? A systematic review
}

Angela Rao a, *, Louise D. Hickman a, David Sibbritt a, b, Phillip

J. Newton a, c,Jane L. Phillips a, c

a Faculty of Health, University of Technology Sydney, Australia

b Australian Research Centre in Complementary and Integrative Medicine (ARCCIM), University of Technology Sydney, Australia

C Centre for Cardiovascular \& Chronic Care, University of Technology Sydney, Australia

ABSTRACT:

Background: Emerging evidence suggests that some people living with non-communicable diseases (NCDs) have integrated energy healing into their self-management strategy, however little is known about its efficacy.

Purpose: To identify energy healing interventions that impacted positively on the symptom management outcomes for patients living in the community with various NCDs.

Methods: A systematic review of energy healing interventions for the management of noncommunicable disease related symptoms, conducted between 01 January 2000 and 21 April 2015, published in an English peer-reviewed journal. This review conforms to the PRISMA statement.

Results: 27 studies were identified that evaluated various energy healing interventions involving $(n=3159)$. Thirteen of the energy healing trials generated statistically significant outcomes.

Conclusions: Energy healing has demonstrated some improvement in illness symptoms, however high level evidence consistently demonstrating efficacy is lacking. Further more robust trials are required to better understand which elements of energy healing interventions are associated with positive outcomes. 


\section{INTRODUCTION}

It is increasingly recognised that many people with non-communicable diseases (NCDs) have unmet needs and novel approaches that address the psychosocial and emotional aspects of symptom management are required [1]. Despite technological advances in the delivery of conventional medical and surgical care, many adults with NCDs in the community find it difficult to effectively manage their symptoms [2,3]. Energy healing is a novel adjunct approach that requires evaluation for its potential to be utilised as a part of interdisciplinary collaborative care [4]. While people affected by NCDs utilise a range of pharmacological treatments there is emerging evidence that some people are integrating energy healing into their self-management strategy $[5,6]$.

Energy healing is defined by the National Centre for Complementary and Integrative Health (NCCIM) as involving "...the channelling of healing energy through the hands of a practitioner into the client's body to restore a normal energy balance and, therefore, health" [7]. Energy healing utilises an expanded paradigm of health and disease, in that it identifies the physical body as being surrounded by an energetic field, with illness arising in part due to psychosocial or emotional issues that manifest as an imbalance in energetic flow [8]. The mechanism of effect of energy healing remains unknown. Energy healing includes both contact healing, which involves light touch on or a few inches above the body, or distance healing, which involves focused intention from a remote location, and has a variety of applications not limited to spiritual healing, Therapeutic Touch, Healing Touch and Reiki. Therapeutic Touch involves a five step process of centering (generation of a quiet, focused state and the intention to heal), assessment of the energy field using the hands to sense energetic cues such as density or blockage or depletion, direction and modulation of energy to balance and restore the energy field, and an evaluation phase to determine the restoration of balance to the energy field of the recipient, which is purported to produce a relaxation effect [9]. A relaxation response refers to positive physiological changes induced by an autonomic response that diminishes the hypothalamic-pituitary-adrenal axis 
response of the autonomic nervous system by reducing norepinephrine release [10]. Healing touch is a similar modality to Therapeutic Touch however purports to have an extended focus that incorporates spiritual growth and intuitive awareness by means of balancing the chakras (seven major energy centres within the body) [11]. Reiki is a method of healing which purports to use universal life force energy, which flows from the practitioner to the areas of the body where it is needed [12]. Reiki generates a sense of internal stillness and peace with sensitisation and perception of energy within the hands, which is then directed to the patient using twelve specific hand positions [13]. This process is referred to as 'attunement'. Energy healing conducted over distance ('distant healing') using intention is believed to be effective because its purported effects are not dissipated or blocked by conventional energy barriers [14]. The variety in energy healing approaches also affects the ways in which contact and non-contact approaches can be adequately assessed. Contact healing approaches require simulation in sham conditions in order to control for the effects of expectation or placebo, whereas this is not required in distance healing where no contact is made between the healer and the recipient.

Healing prioritises psychological and spiritual components of health in a way that conventional health care is currently not able to adequately address, encompassing the broader medical, social and emotional contexts of illness as inherent in the treatment plan [15]. These therapies may be particularly attractive as an adjunct to conventional treatments, where the condition is particularly unresponsive to allopathic approaches or reflects a chronic condition for which changes in wellbeing may be as beneficial as effecting actual biological change. For example, the capacity of adults with NCDs to effectively self-manage their illness symptoms requires an understanding of the relationship between one's emotional state and personality traits with the capacity to cope with life stressors [12]. Despite energy healing increasingly being used as part of an overall self-management strategy for symptoms associated with various NCDs [16], there is inconclusive evidence these type of interventions improved the well-being of adults with a terminal illness [17]. The numbers of adults using healing therapies is difficult to determine, with estimates varying between $0.7 \%$ and $24.4 \%$ in Western countries $[18,19]$. 
Whilst use of healing has been found to be higher among adults managing NCDs it is unclear as to how many adults with NCDs actually use healing as part of a self-management strategy [5].

Four systematic reviews published at of start of the new millennium, investigated the impact of healing on disease or illness symptoms ( $n=3102 ; 22$ trials) [20], medical conditions ( $n=2774 ; 23$ trials) [21], or clinical conditions ( $n=8455 ; 45$ studies) [22] in hospital settings and the community. Each of these systematic reviews generated inconclusive results due to heterogeneity of comparison groups and/or poor study design. Issues related to study design included: inadequate reporting of group baseline comparability, inadequate or lack of blinding, lack of sensitivity of measurements used (distant healing); lack of reliability, study power; omission of confidence intervals, lack of independent replication and increased risk of type II errors [20-22].

Over the past decade further work in this area has generated small, significant improvements in healing yet remain overall inconclusive [23-25]. This includes a Cochrane review of depression and anxiety [25], and two other systematic reviews that demonstrated improvements in general well-being [23], pain intensity, negative behavioural symptoms and anxiety [24], however none of the reviews have focused exclusively on outpatients with non-communicable diseases. This review seeks to fill this gap in the literature by focusing on adults living in the community that use energy healing as a NCD selfmanagement strategy.

\section{Aims}

This systematic review aims to: appraise randomised control trials (RCT) results of energy healing interventions designed to improve outcomes for people living with various non-communicable diseases; and identify and classify the key elements of the effective energy healing interventions that have demonstrated ability to enhance outcomes for patients with symptoms associated with various NCDs.

\section{METHODS}

\section{Information Sources and Search}


This systematic review was completed according to the PRISMA 2009 Checklist. Databases searched included AMED, MEDLINE, PUBMED, CINAHL and the Cochrane Database of Systematic Reviews from 01 January 2000 to 'current' last searched on 21st April 2015. Medical Subject Headings (MeSH) and keyword search terms were used for the following search terms: 'Prayer' OR 'Spiritual Healing', AND ‘Diabetes Mellitus’ OR ‘Chronic Disease’ OR ‘Heart Disease’ OR ‘Asthma’ OR ‘Pulmonary Disease, Chronic Obstructive' OR, 'Osteoarthritis' OR 'Arthritis, Rheumatoid' OR' Neoplasms' OR ‘Anxiety' OR 'Depression'. The search was expanded to incorporate keywords and MeSH including 'Therapeutic Touch', 'Healing Touch' or 'Reiki', which are the three most commonly utilised energy healing modalities. Limits included English language, and a filter of 'clinical trials' and 'randomised controlled trials' was applied to the PUBMED search. Additional limits of 'peer reviewed', and 'human' were applied to the CINAHL search. Other sources included the International Society of Subtle Energies and Energy Medicine Database and reference lists of included papers were searched for additional articles. Authors of included papers were contacted via email as required.

\section{Eligibility Criteria}

Populations for inclusion in this review were adults with non-communicable diseases including heart disease, diabetes, asthma or COPD, rheumatoid arthritis or osteoarthritis, cancer, depression or anxiety that were living in the community. Populations clearly defined as diagnosed chronic illnesses and/or included one or more of the above NCDs were eligible for inclusion. Energy healing interventions included distance healing and contact healing techniques (that involve light touch or no touch). Energy healing modalities included spiritual healing, Therapeutic Touch, Healing Touch and Reiki. Studies required a separate comparison group, a usual care control group, or a placebo or sham control group or expectancy control group to be eligible for inclusion. All outcomes were considered. Non-English articles, dissertations, and studies involving paediatric populations were also excluded.

\section{Study selection}


All phase II and phase III RCTs, and pilot studies assessing the impact of energy healing studies that adhered to the definition of energy healing among outpatient populations with NCDs were included. Phase III studies were defined as fully powered RCTs that included a clearly stated primary outcome measure, and a sample size calculation that was powered to the primary outcome. Phase II studies and pilot studies were suitable for inclusion if the studies included randomisation procedures and a control and/or comparison group.

\section{Data extraction and quality assessment}

The title and abstract were screened for eligibility and all duplicates were removed (AR). Discrepancies regarding inclusion and exclusion were resolved by consensus (AR, JLP). Bias was determined in accordance with the Cochrane 'Risk of Bias Table' [26].

\section{Data synthesis}

The wide variability in the different NCD populations, elements of energy healing and primary outcome measures prevented a meta-analysis from being undertaken. Thematic analysis of the interventions, outcome measures and results have been synthesised into a narrative review.

\section{RESULTS:}

\section{Study selection}

The initial search generated 2327 articles. After duplicate papers were removed ( $\mathrm{n}=158$ studies), 2169 abstracts were screened. Following a process of review, elimination and hand searching, 27 studies were identified (Figure 1). 


\section{Figure 1: Study selection}

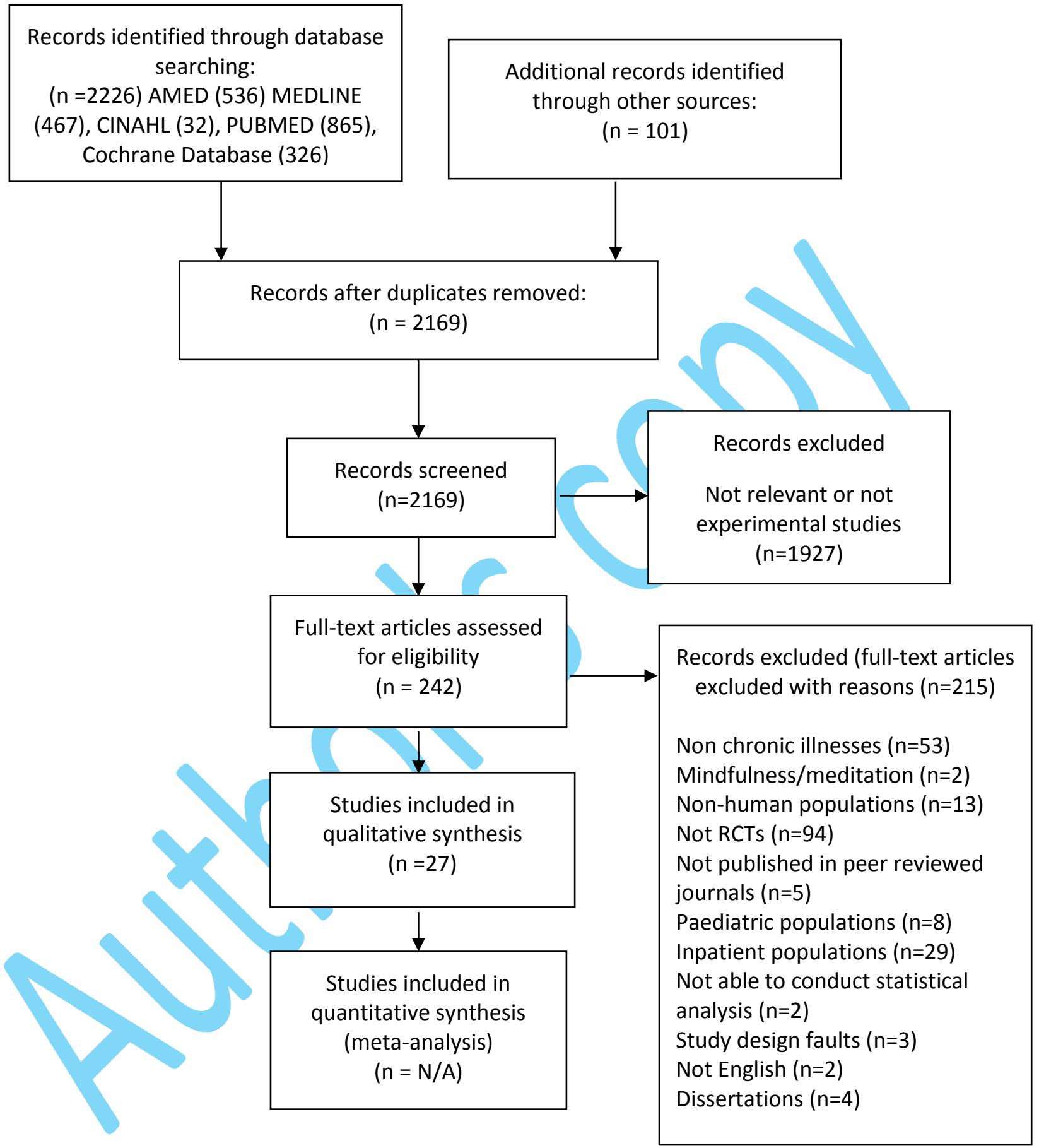




\section{Study characteristics}

Half of the studies were conducted in the US or UK $(n=18)$ with the remainder conducted in other developed nations. Spiritual healing was used in 9 studies, Therapeutic Touch was use in four studies, Healing Touch was used in four studies, Reiki was used in 8 studies and Johnston's technique was used in one study. The mean number of participants/study was $117(S D \pm 159)$ and two thirds were female (75\%). Participants included people living in the community with: arthritic disease ( $n=277 ; 8$ studies), various cancer ( $n=317 ; 7$ studies), depression or anxiety $(n=54 ; 2$ studies), chronic pain ( $n=$ $267 ; 7$ studies), hypertension ( $n=115 ; 1$ studies), chronic illnesses ( $n=648 ; 3$ studies), asthma ( $n=92$; 1 study) and diabetes ( $n=207 ; 1$ study) (Table 1 ).

The duration of energy healing interventions ranged from five weeks to 28 months overall, and intensity ranged from 15 minutes to 90 minutes overall. Energy healers had diverse affiliations or were affiliated with local healing organisations. 
Table 1. Summary table of included energy healing interventions

\begin{tabular}{|c|c|c|c|c|c|c|c|c|c|c|c|c|}
\hline \multirow{2}{*}{$\begin{array}{l}\text { Authorl } \\
\text { country }\end{array}$} & \multirow{2}{*}{$\begin{array}{l}\text { Study } \\
\text { design }\end{array}$} & \multirow{2}{*}{$\begin{array}{l}\text { Primary } \\
\text { outcomes }\end{array}$} & \multirow[t]{2}{*}{ Population } & \multirow[t]{2}{*}{ Number } & \multicolumn{3}{|c|}{ Intervention content } & \multirow{2}{*}{\begin{tabular}{|l|}
$\begin{array}{l}\text { Expectancy } \\
\text { measures }\end{array}$ \\
\end{tabular}} & \multirow{2}{*}{\begin{tabular}{|l|} 
Intensityl \\
Duration
\end{tabular}} & \multirow{2}{*}{$\begin{array}{l}\text { Control } \\
\text { group }\end{array}$} & \multirow[t]{2}{*}{ Outcome measures } & \multirow[t]{2}{*}{ Outcomes } \\
\hline & & & & & Modality & \begin{tabular}{|l|} 
Distance \\
or contact \\
healing
\end{tabular} & Blinding procedure(s) & & & & & \\
\hline \multirow[t]{2}{*}{$\begin{array}{l}\text { Beutler, } \\
\text { Attevelt [27] } \\
\text { Netherlands }\end{array}$} & $\begin{array}{l}\text { Prospective } \\
\text { RCT }\end{array}$ & hypertension & $\begin{array}{l}\text { Hypertension } \\
(\mathrm{SBP} \geq 140 \\
\mathrm{mmHg} ; \\
\mathrm{DBP} \geq 90 \\
\mathrm{mmHg})\end{array}$ & $\begin{array}{l}115 \\
\text { Distance = } 37 \\
\text { Sham = 38 }\end{array}$ & $\begin{array}{l}\text { Spiritual } \\
\text { healing }\end{array}$ & Distance & $\begin{array}{l}\text { Healing and Sham groups } \\
\text { separated from healer by a } \\
\text { one way screen, monitored } \\
\text { by CCTV }\end{array}$ & No & $\begin{array}{l}\text { I: } 20 \text { mins } \\
\text { D: weekly x } \\
15 \text { weeks }\end{array}$ & Sham & $\begin{array}{l}\text { SBP, DBP at baseline, } \\
\text { pre and post intervention }\end{array}$ & $\begin{array}{l}\text { No improvement in } \\
\text { hypertension. }\end{array}$ \\
\hline & & & & Contact $=40$ & & Contact & No blinding & & & & & $\begin{array}{l}\text { Increased DBP post } \\
\text { contact healing } \\
(p<0.05)\end{array}$ \\
\hline $\begin{array}{l}\text { Redner, } \\
\text { Briner [28] } \\
\text { US }\end{array}$ & \begin{tabular}{|l}
$2 \times 3 \times 2$ \\
repeated \\
measures \\
design
\end{tabular} & Chronic pain & $\begin{array}{l}\text { Chronic } \\
\text { arthritis, } \\
\text { headache, } \\
\text { low back pain }\end{array}$ & $\begin{array}{l}47 \\
\text { Healing= } 23 \\
\text { Attention } \\
\text { control= } 24\end{array}$ & $\begin{array}{l}\text { Johnston's } \\
\text { technique }\end{array}$ & $\begin{array}{l}\text { Contact } \\
\text { (no } \\
\text { touch) }\end{array}$ & $\begin{array}{l}\text { Actor mimicking hand } \\
\text { movement }\end{array}$ & No & $\begin{array}{l}\text { I: } 30 \text { mins } \\
\text { D: four } \\
\text { occasions }\end{array}$ & $\begin{array}{l}\text { Attention } \\
\text { Control }\end{array}$ & $\begin{array}{l}\text { Cornell Medical Index, } \\
\text { McGill Melzack Pain } \\
\text { Questionnaire, POMS } \\
\text { (weekly), physical } \\
\text { medical check (session } 1 \\
\text { \&6), pain diary }\end{array}$ & $\begin{array}{l}\text { No improvement in } \\
\text { physical condition or } \\
\text { mood; increased } \\
\text { anxiety }(p<0.05) ; \\
\text { decreased sensory } \\
\text { and affective pain } \\
\text { (both } p<0.05 \text { ) }\end{array}$ \\
\hline $\begin{array}{l}{[29]} \\
\text { Finland }\end{array}$ & $\begin{array}{l}\text { Pilot } \\
\text { study }\end{array}$ & $\begin{array}{l}\text { Pain, } \\
\text { depression, } \\
\text { psychological } \\
\text { distress, } \\
\text { coping, health } \\
\text { locus of } \\
\text { control }\end{array}$ & $\begin{array}{l}\text { Idiopathic } \\
\text { chronic pain } \\
\geq 6 \text { months } \\
\text { duration }\end{array}$ & $\begin{array}{l}24 \\
\text { Healing = } 12 \\
\text { Control }=12\end{array}$ & Spir & $\mathrm{COI}$ & No blinding & No & $\begin{array}{l}\text { I: } 40 \text { mins } \\
\text { D: } 3-8 \\
\text { sessions as } \\
\text { per healer }\end{array}$ & No & $\begin{array}{l}\text { VAS, Pain Clinic } \\
\text { Investigation Formula, } \\
\text { IASP Database Outline, } \\
\text { Hopkin's Symptom } \\
\text { Checklist, MHQ, BDI, } \\
\text { Coping Strategy } \\
\text { Questionnaire at } \\
\text { baseline, } 2 \text { weeks. } \\
\text { IASP at one year }\end{array}$ & $\begin{array}{l}\text { No reduction in pain, } \\
\text { depression or distress. } \\
\text { Reduced } \\
\text { hopelessness (coping) } \\
\text { ( } p<0.05 \text { ), Improved } \\
\text { acceptance of } \\
\text { psychological } \\
\text { contributors to pain } \\
\text { (locus of control) } \\
(p<0.05)\end{array}$ \\
\hline $\begin{array}{l}\text { Eckes Peck } \\
{[30]} \\
\text { US }\end{array}$ & \begin{tabular}{|l} 
Two group \\
longitudinal \\
repeated \\
measures \\
design
\end{tabular} & Pain, distress & $\begin{array}{l}\text { Non- } \\
\text { institutionalised } \\
\text { older adults } \\
\text { with } \\
\text { degenerative }\end{array}$ & $\begin{array}{l}108 \\
\text { Healing }=60 \\
\text { PMR }=48\end{array}$ & $\begin{array}{l}\text { Therapeutic } \\
\text { Touch }\end{array}$ & Contact & $\begin{array}{l}\text { Actor used to mimic } \\
\text { movements }\end{array}$ & No & $\begin{array}{l}\text { I: } 10-33 \text { mins } \\
\text { D: } 6 \text { weeks }\end{array}$ & \begin{tabular}{|l} 
Subjects \\
served as \\
own \\
controls in \\
baseline no
\end{tabular} & VAS (pain, distress) & $\begin{array}{l}\text { No significant } \\
\text { improvement in pain or } \\
\text { distress compared to } \\
\text { progressive muscle } \\
\text { relaxation }\end{array}$ \\
\hline
\end{tabular}




\begin{tabular}{|c|c|c|c|c|c|c|c|c|c|c|c|c|}
\hline \multirow{2}{*}{$\begin{array}{l}\text { Authorl } \\
\text { country }\end{array}$} & \multirow{2}{*}{$\begin{array}{l}\text { Study } \\
\text { design }\end{array}$} & \multirow{2}{*}{\begin{tabular}{|l|} 
Primary \\
outcomes
\end{tabular}} & \multirow[t]{2}{*}{ Population } & \multirow[t]{2}{*}{ Number } & \multicolumn{3}{|c|}{ Intervention content } & \multirow{2}{*}{\begin{tabular}{|l|}
$\begin{array}{l}\text { Expectancy } \\
\text { measures }\end{array}$ \\
\end{tabular}} & \multirow{2}{*}{\begin{tabular}{|l|} 
Intensityl \\
Duration \\
\end{tabular}} & \multirow{2}{*}{\begin{tabular}{|l|} 
Control \\
group
\end{tabular}} & \multirow[t]{2}{*}{ Outcome measures } & \multirow[t]{2}{*}{ Outcomes } \\
\hline & & & & & Modality & \begin{tabular}{|l|} 
Distance \\
or contact \\
healing
\end{tabular} & Blinding procedure(s) & & & & & \\
\hline & & & $\begin{array}{l}\text { arthritis, }>6 \\
\text { months chronic } \\
\text { pain }\end{array}$ & & & & & & & & & \\
\hline $\begin{array}{l}\text { Gordon, } \\
\text { Merenstein } \\
{[31]}\end{array}$ & $\begin{array}{l}\text { Pilot } \\
\text { study }\end{array}$ & $\begin{array}{l}\text { pain, } \\
\text { general } \\
\text { well-being, } \\
\text { health } \\
\text { status }\end{array}$ & Osteoarthritis & $\begin{array}{l}31 \\
\text { Healing = } 8 \\
\text { Sham = } 11 \\
\text { Control = } 8\end{array}$ & $\begin{array}{l}\text { Therapeutic } \\
\text { Touch }\end{array}$ & Contact & $\begin{array}{l}\text { Actor mimicking hand } \\
\text { movements }\end{array}$ & No & $\begin{array}{l}\text { I: not stated } \\
\text { D: weekly x } \\
6 \text { weeks }\end{array}$ & $\begin{array}{l}\text { No } \\
\text { contact } \\
\text { with } \\
\text { investigat } \\
\text { ors }\end{array}$ & $\begin{array}{l}\text { Stanford HAQ, West } \\
\text { Haven Yale MPI at } \\
\text { baseline, week 7, 13; } \\
\text { VAS (pain, general well- } \\
\text { being) baseline, pre and } \\
\text { post sessions, week 7, } \\
\text { 13, in depth interview } \\
\text { week } 7 .\end{array}$ & $\begin{array}{l}\text { Decreased pain and } \\
\text { improved function } \\
\text { (MPI); } \\
\text { No improvement in } \\
\text { general health, health } \\
\text { status. }\end{array}$ \\
\hline $\begin{array}{l}\text { Dressen } \\
\text { and Singg } \\
{[12]} \\
\text { US }\end{array}$ & $\begin{array}{l}\text { Pilot } \\
\text { study }\end{array}$ & $\begin{array}{l}\text { pain, } \\
\text { depression, } \\
\text { anxiety, self } \\
\text { esteem }\end{array}$ & $\begin{array}{l}\text { Chronically ill, } \\
\text { symptomatic, } \\
\text { pain }\end{array}$ & $\begin{array}{l}120 \\
\text { PMR }=30 \\
\text { Reiki }=30 \\
\text { Sham }=30 \\
\text { Control }=30\end{array}$ & Reiki & Contact & $\begin{array}{l}\text { Actor mimicking hand } \\
\text { movements }\end{array}$ & No & $\begin{array}{l}\text { I: } 30 \text { mins } \\
\text { D: } 10 \\
\text { biweekly } \\
\text { sessions }\end{array}$ & $\begin{array}{l}\text { Sat in } \\
\text { office } \\
\text { with } \\
\text { reading } \\
\text { material } \\
\text { of their } \\
\text { choice }\end{array}$ & $\begin{array}{l}\text { Pain rating intensity } \\
\text { (affective and total), } \\
\text { Social Readjustment } \\
\text { Rating Scale, MGPQ, BDI } \\
\text { II, STAI, Rotter I-E Scale, } \\
\text { Rosenberg Self Esteem } \\
\text { Scale, Belief in Personal } \\
\text { Control Scale }\end{array}$ & $\begin{array}{l}\text { Reduced pain intensity } \\
\left(p=0.0001, w^{2}=0.18\right) \\
\text { sensory pain rating } \\
\left(p=0.03 ; w^{2}=.05\right), \\
\text { evaluative pain rating } \\
\left(p=0.001, w^{2}=.13\right) . \\
\text { Affective and total PRI } \\
\text { not significantly reduced. } \\
\text { Reduced (depression } \\
\left(p=0.0001, w^{2}=.34\right), \text { state } \\
\text { and trait anxiety (both } \\
p=0.0001 ; w^{2}=.28, .29 \\
\text { respectively). } \\
\text { Improved self-esteem } \\
\left(p=0.2, w^{2}=.05\right)\end{array}$ \\
\hline $\begin{array}{l}\text { Lin and } \\
\text { Taylor [32] } \\
\text { US }\end{array}$ & $\begin{array}{l}\text { Pre-post } \\
\text { test }\end{array}$ & $\begin{array}{l}\text { pain, } \\
\text { anxiety, } \\
\text { salivary } \\
\text { coritsol }\end{array}$ & $\begin{array}{l}\text { Chronic } \\
\text { pain, } \\
\text { anxiety, } \\
\text { older adults }\end{array}$ & $\begin{array}{l}95 \\
\text { Healing }=31 \\
\text { Sham }=29 \\
\text { Control }=30\end{array}$ & $\begin{array}{l}\text { Therapeutic } \\
\text { Touch }\end{array}$ & Contact & $\begin{array}{l}\text { Actor mimicking hand } \\
\text { movements }\end{array}$ & No & $\begin{array}{l}\text { I: } 30 \text { mins } \\
\text { D: } 3 \\
\text { consecutive } \\
\text { days }\end{array}$ & $\begin{array}{l}\text { Nursing } \\
\text { home } \\
\text { care }\end{array}$ & $\begin{array}{l}\text { Numeric pain rating } \\
\text { scale, STAI (Y-1), } \\
\text { radioimmunoassay 5-10 } \\
\text { minutes pre and post } \\
\text { sessions }\end{array}$ & $\begin{array}{l}\text { Significantly reduced } \\
\text { pain rating }(p<0.001) ; \\
\text { anxiety }(p<0.01) ; \text { no } \\
\text { reduction in cortisol }\end{array}$ \\
\hline
\end{tabular}




\begin{tabular}{|c|c|c|c|c|c|c|c|c|c|c|c|c|}
\hline \multirow{2}{*}{\begin{tabular}{|l|} 
Authorl \\
country \\
\end{tabular}} & \multirow{2}{*}{\begin{tabular}{|l|} 
Study \\
design \\
\end{tabular}} & \multirow{2}{*}{\begin{tabular}{|l|} 
Primary \\
outcomes \\
\end{tabular}} & \multirow[t]{2}{*}{ Population } & \multirow[t]{2}{*}{ Number } & \multicolumn{3}{|c|}{ Intervention content } & \multirow{2}{*}{\begin{tabular}{|l|}
$\begin{array}{l}\text { Expectancy } \\
\text { measures }\end{array}$ \\
\end{tabular}} & \multirow{2}{*}{\begin{tabular}{|l|} 
Intensityl \\
Duration
\end{tabular}} & \multirow{2}{*}{$\begin{array}{l}\text { Control } \\
\text { group }\end{array}$} & \multirow[t]{2}{*}{ Outcome measures } & \multirow[t]{2}{*}{ Outcomes } \\
\hline & & & & & Modality & \begin{tabular}{|l|} 
Distance \\
or contact \\
healing \\
\end{tabular} & Blinding procedure(s) & & & & & \\
\hline $\begin{array}{l}\text { le Gallez, } \\
\text { Dimmock } \\
\text { [33] } \\
\text { UK }\end{array}$ & $\begin{array}{l}\text { Phase II } \\
\text { parallel } \\
\text { group }\end{array}$ & $\begin{array}{l}\text { Pain } \\
\text { perception }\end{array}$ & $\begin{array}{l}\text { Active } \\
\text { rheumatoid } \\
\text { arthritis on } \\
\text { DMARD }\end{array}$ & $\begin{array}{l}29 \\
\text { Healing }=15 \\
\text { Control }=14\end{array}$ & $\begin{array}{l}\text { Spiritual } \\
\text { healing }\end{array}$ & $\begin{array}{l}\text { Contact } \\
\text { (no } \\
\text { touch) }\end{array}$ & no & no & $\begin{array}{l}\text { I: } 20-120 \text { mins } \\
\text { D: weekly as } \\
\text { desired (13-24 } \\
\text { sessions) x } 24 \\
\text { weeks }\end{array}$ & $\begin{array}{l}\text { Doctor } \\
\text { visit }\end{array}$ & $\begin{array}{l}\text { Grip strength, Summated } \\
\text { Change Scores, } \mathrm{RAl}^{*}, \\
\text { pain score, bloods EMS*, } \\
\mathrm{MHQ}^{*} \mathrm{SHAQ}{ }^{*}, \mathrm{EPQ}{ }^{*} \text { at } \\
\text { baseline, week } 8,16 \text { and } \\
24 \text { weeks }\end{array}$ & $\begin{array}{l}\text { No improvement in } \\
\text { arthritis symptoms or } \\
\text { biochemical markers } \\
\text { of arthritis }\end{array}$ \\
\hline \multirow[t]{2}{*}{$\begin{array}{l}\text { Abbot, } \\
\text { Harkness } \\
{[34]}\end{array}$} & $\begin{array}{l}\text { Phase III } \\
\text { parallel } \\
\text { group } \\
\text { design }\end{array}$ & $\begin{array}{l}\text { Total pain } \\
\text { rating } \\
\text { index } \\
\text { score }\end{array}$ & $\begin{array}{l}\text { Chronic pain } \\
>6 \text { months } \\
\text { duration }\end{array}$ & $\begin{array}{l}126 \\
\text { Distance = } 28 \\
\text { Sham }=27\end{array}$ & $\begin{array}{l}\text { Spiritual } \\
\text { healing }\end{array}$ & Distance & $\begin{array}{l}\text { Healer sat inside a cabinet } \\
\text { with one way mirror (healer } \\
\text { to recipient) } \\
\text { Sham - Empty cabinet, } \\
\text { chair moved intermittently to } \\
\text { mimic person inside }\end{array}$ & $Y_{\rho \rho}$ & $\begin{array}{l}\text { I: } 30 \text { mins; } \\
\text { D: weekly x } \\
8 \text { weeks } \\
\text { over a } 10 \\
\text { week period }\end{array}$ & Sham & $\begin{array}{l}\text { MPQ, at baseline, pre } \\
\text { first treatment, post } \\
\text { treatment at } 4 \text { weeks } \\
\text { and post study at } 10 \\
\text { weeks. }\end{array}$ & $\begin{array}{l}\text { No reduction in total } \\
\text { pain rating index } \\
\text { (MPQ) }\end{array}$ \\
\hline & & & & $\begin{array}{l}\text { Contact }=25 \\
\text { Sham }=25\end{array}$ & & Contact & $\begin{array}{l}\text { Actor }{ }^{*} \text { mimicking hand } \\
\text { movements }\end{array}$ & & & Sham & & \\
\hline $\begin{array}{l}\text { Wiesendanger, } \\
\text { Werthmüller } \\
{[35]} \\
\text { Switzerland/ } \\
\text { Germany }\end{array}$ & \begin{tabular}{|l} 
Phase III \\
parallel \\
group \\
design
\end{tabular} & $\begin{array}{l}\text { Quality of } \\
\text { life }\end{array}$ & $\begin{array}{l}\text { Diagnosed } \\
\text { chronic } \\
\text { illness } \\
\text { minimum } 1 \\
\text { year duration }\end{array}$ & $\begin{array}{l}119 \\
\text { Distance }=30 \\
\text { Amulet }=10 \\
\text { Contact }=20 \\
\text { Control }=59\end{array}$ & $\begin{array}{l}\text { Spiritual } \\
\text { healing }\end{array}$ & Distance & $\begin{array}{l}\text { Separated by country (no } \\
\text { blinding) } \\
\text { Distance-anonymous } \\
\text { healers } \\
\text { Amulet-wore bracelet that } \\
\text { had healing energy directed } \\
\text { to it } \\
\text { Contact -allowed phone } \\
\text { contact prior to intervention } \\
\text { after allocation } \\
\end{array}$ & Yes & $\begin{array}{l}\text { I: healer } \\
\text { determined } \\
\text { (average } 23 \\
\text { mins, range } \\
5 \text { mins-2 } \\
\text { hrs); } \\
\text { D: twice } \\
\text { weekly x } 5 \\
\text { months }\end{array}$ & waitlist & $\begin{array}{l}\text { SF-36 at baseline and } \\
\text { post intervention. }\end{array}$ & $\begin{array}{l}\text { Improved quality of life } \\
(p<0.00045, d=0.66) .\end{array}$ \\
\hline $\begin{array}{l}\text { Woods, } \\
\text { Arnstein } \\
{[36]} \\
\text { US }\end{array}$ & $\begin{array}{l}\text { Pilot } \\
\text { study }\end{array}$ & \begin{tabular}{|l|} 
Pain, \\
distress, self \\
efficacy, \\
disability, \\
power
\end{tabular} & Chronic pain & $\begin{array}{l}12 \\
\text { Healing } \\
\text { Control }\end{array}$ & $\begin{array}{l}\text { Therapeutic } \\
\text { Touch (and } \\
\text { relaxation } \\
\text { response) }\end{array}$ & Contact & No blinding & No & $\begin{array}{l}\text { I: healer } \\
\text { determined } \\
\text { D:weekly x } 3 \\
\text { weeks, then } \\
9 \text { weeks } \\
\text { CBT } \\
\end{array}$ & $\begin{array}{l}\text { Relaxation } \\
\text { response }\end{array}$ & $\begin{array}{l}\text { Pain disability Index, VAS } \\
\text { (pain, distress), PKPCT*, } \\
\text { PDI, CPSS, participant } \\
\text { diary }\end{array}$ & $\begin{array}{l}\text { No significant } \\
\text { improvement in pain, } \\
\text { distress self-efficacy, } \\
\text { disability, power. }\end{array}$ \\
\hline $\begin{array}{l}\text { Post-White, } \\
\text { Kinney [37] }\end{array}$ & \begin{tabular}{|l|} 
Phase III \\
crossover \\
design
\end{tabular} & $\begin{array}{l}\text { Fatigue, } \\
\text { anxiety, } \\
\text { mood }\end{array}$ & cancer & $\begin{array}{l}239 \\
\text { Healing }=77 \\
\text { Massage }=78\end{array}$ & $\begin{array}{l}\text { Healing } \\
\text { Touch }\end{array}$ & Contact & $\begin{array}{l}\text { No blinding } \\
\text { Therapeutic Massage or } \\
\text { Presence (soft music) }\end{array}$ & No & $\begin{array}{l}\text { I: } 45 \text { mins; } \\
\text { D: weekly x } \\
4 \text { weeks }\end{array}$ & $\begin{array}{l}\text { Standard } \\
\text { cancer } \\
\text { treatment }\end{array}$ & $\begin{array}{l}\text { POMS at weeks } 1,4,5 \text {, } \\
\text { and } 8\end{array}$ & $\begin{array}{l}\text { Reduced mood } \\
\text { disturbance }(p=0.003) \\
\text { and fatigue }(p=0.023)\end{array}$ \\
\hline
\end{tabular}




\begin{tabular}{|c|c|c|c|c|c|c|c|c|c|c|c|c|}
\hline \multirow{2}{*}{$\begin{array}{l}\text { Authorl } \\
\text { country }\end{array}$} & \multirow{2}{*}{\begin{tabular}{|l|} 
Study \\
design
\end{tabular}} & \multirow{2}{*}{\begin{tabular}{|l|} 
Primary \\
outcomes \\
\end{tabular}} & \multirow[t]{2}{*}{ Population } & \multirow[t]{2}{*}{ Number } & \multicolumn{3}{|c|}{ Intervention content } & \multirow{2}{*}{\begin{tabular}{|l|}
$\begin{array}{l}\text { Expectancy } \\
\text { measures }\end{array}$ \\
\end{tabular}} & \multirow{2}{*}{\begin{tabular}{|l|} 
Intensityl \\
Duration \\
\end{tabular}} & \multirow{2}{*}{\begin{tabular}{|l|} 
Control \\
group \\
\end{tabular}} & \multirow[t]{2}{*}{ Outcome measures } & \multirow[t]{2}{*}{ Outcomes } \\
\hline & & & & & Modality & \begin{tabular}{|l|} 
Distance \\
or contact \\
healing \\
\end{tabular} & Blinding procedure(s) & & & & & \\
\hline US & & disturbance & & Presence $=75$ & & & $\begin{array}{l}\text { Conversation allowed for all } \\
\text { groups }\end{array}$ & & & & & $\begin{array}{l}\text { vs usual care. } \\
\text { No improvement in } \\
\text { anxiety. }\end{array}$ \\
\hline $\begin{array}{l}\text { Cook, } \\
\text { Guerrerio } \\
{[38]}\end{array}$ & $\begin{array}{l}\text { RCT } \\
\text { Phase II }\end{array}$ & $\begin{array}{l}\text { Health } \\
\text { related } \\
\text { quality of } \\
\text { life }\end{array}$ & $\begin{array}{l}\text { Cancer } \\
\text { patients } \\
\text { receiving } \\
\text { outpatient } \\
\text { radiotherapy }\end{array}$ & $\begin{array}{l}62 \\
\text { Healing }=34 \\
\text { Sham }=28\end{array}$ & $\begin{array}{l}\text { Healing } \\
\text { Touch }\end{array}$ & Contact & $\begin{array}{l}\text { Actor mimicking hand } \\
\text { movements }\end{array}$ & No & $\begin{array}{l}\text { I: } 30 \text { mins } \\
\text { D: } 6 \\
\text { sessions } \times 4 \\
\text { weeks }\end{array}$ & Sham & $\begin{array}{l}\text { SF-36 at baseline and } \\
\text { post intervention }\end{array}$ & $\begin{array}{l}\text { Significantly improved } \\
\text { quality of life }(p<0.06) \\
\text { overall, and improved } \\
\text { pain }(p<0.02) \text { vitality } \\
\text { ( } p<0.03) \text { and physical } \\
\text { functioning }(p<0.05) \text {. }\end{array}$ \\
\hline $\begin{array}{l}\text { Goldman } \\
\text { Shore [39] } \\
\text { US }\end{array}$ & $\begin{array}{l}3 \times 3 \\
\text { factorial } \\
\text { design }\end{array}$ & $\begin{array}{l}\text { Psychologic } \\
\text { al distress, } \\
\text { depressive } \\
\text { symptoms }\end{array}$ & $\begin{array}{l}\text { Depression, } \\
\text { MS, borderline } \\
\text { personality, } \\
\text { mood or } \\
\text { anxiety } \\
\text { disorders, } \\
\text { chronic } \\
\text { fatigue, } \\
\text { fibromyalgia, } \\
\text { non-terminal } \\
\text { cancer }\end{array}$ & $\begin{array}{l}45 \\
\text { Reiki }=16 \\
\text { Sham }=16\end{array}$ & Reiki & Distance & $\begin{array}{l}\text { Distance Reiki } \\
\text { administered off site } \\
\text { Distance Sham Reiki-- } \\
\text { participants believed they } \\
\text { were receiving Reiki, but did } \\
\text { not. }\end{array}$ & no & $\begin{array}{l}\text { I: } 1-1.5 \mathrm{hrs} \\
\text { D: } 6 \text { weeks }\end{array}$ & $\begin{array}{l}\text { Distance } \\
\text { Sham } \\
\text { Reiki }\end{array}$ & $\begin{array}{l}\text { BDI, Beck Hopelessness } \\
\text { Scale, PSS pre and } \\
\text { post-test, } 1 \text { year follow } \\
\text { up }\end{array}$ & $\begin{array}{l}\text { Reduced perceived } \\
\text { stress (Reiki } p=0.004 ; \\
\text { distance Reiki } p=0.005 \text { ); } \\
\text { depressive symptoms } \\
\text { (Reiki } p=0.05 \text {; distance } \\
\text { Reiki } p=0.004 \text { ); } \\
\text { hopelessness (Reiki } \\
p=0.02 \text {; distance Reiki } \\
p=0.01 \text { ) vs placebo that } \\
\text { persisted after } 1 \text { year }\end{array}$ \\
\hline $\begin{array}{l}\text { Cleland, } \\
\text { Price [40] } \\
\text { Scotland }\end{array}$ & $\begin{array}{l}\text { Phase III } \\
\text { RCT }\end{array}$ & $\begin{array}{l}\text { Asthma } \\
\text { related } \\
\text { quality of } \\
\text { life }\end{array}$ & $\begin{array}{l}\text { Asthma }>1 \\
\text { year } \\
\text { pharmacolog } \\
\text { ical } \\
\text { treatment }\end{array}$ & $\begin{array}{l}92 \\
\text { healing }=27 \\
\text { sham }=30 \\
\text { control }=31\end{array}$ & $\begin{array}{l}\text { Spiritual } \\
\text { healing }\end{array}$ & $\begin{array}{l}\text { Contact } \\
\text { with guided } \\
\text { visualisatio } \\
n\end{array}$ & $\begin{array}{l}\text { Actor to mimic hand } \\
\text { movements }\end{array}$ & No & $\begin{array}{l}\text { I: } 40 \text { mins } \\
\text { D: weekly x } \\
5 \text { weeks }\end{array}$ & $\begin{array}{l}\text { Not } \\
\text { defined }\end{array}$ & $\begin{array}{l}\text { Juniper AQLQ at } \\
\text { baseline, weeks 2, 4, 8, } \\
12 \text { and } 26 .\end{array}$ & $\begin{array}{l}\text { No reduction in } \\
\text { asthma QOL }\end{array}$ \\
\hline $\begin{array}{l}\text { Gillespie, } \\
\text { Gillespie } \\
{[41]} \\
\text { UK }\end{array}$ & \begin{tabular}{|l|} 
Phase II \\
parallel \\
group \\
RCT
\end{tabular} & Pain & $\begin{array}{l}\text { Type } 2 \\
\text { diabetes, } \\
\text { painful } \\
\text { diabetic } \\
\text { neuropathy }\end{array}$ & $\begin{array}{l}207 \\
\text { Reiki }=93 \\
\text { Sham }=88 \\
\text { Control }=26\end{array}$ & & Contact & $\begin{array}{l}\text { Actors imitated Reiki } \\
\text { practice }\end{array}$ & No & $\begin{array}{l}\text { I: } 25 \text { mins } \\
\text { D: twice } \\
\text { weekly } \times 1 \text { st } \\
\text { week, } \\
\text { weekly x } 12 \\
\text { weeks } \\
\text { thereafter }\end{array}$ & $\begin{array}{l}\text { Not } \\
\text { defined }\end{array}$ & $\begin{array}{l}\text { MPQ at baseline, } 12 \\
\text { weeks }\end{array}$ & $\begin{array}{l}\text { No significant } \\
\text { improvement in pain in } \\
\text { Reiki group vs sham } \\
\text { Reiki group. }\end{array}$ \\
\hline
\end{tabular}




\begin{tabular}{|c|c|c|c|c|c|c|c|c|c|c|c|c|}
\hline \multirow{2}{*}{$\begin{array}{l}\text { Authorl } \\
\text { country }\end{array}$} & \multirow{2}{*}{\begin{tabular}{|l|} 
Study \\
design
\end{tabular}} & \multirow{2}{*}{$\begin{array}{l}\text { Primary } \\
\text { outcomes }\end{array}$} & \multirow[t]{2}{*}{ Population } & \multirow[t]{2}{*}{ Number } & \multicolumn{3}{|c|}{ Intervention content } & \multirow{2}{*}{\begin{tabular}{|l|}
$\begin{array}{l}\text { Expectancy } \\
\text { measures }\end{array}$ \\
\end{tabular}} & \multirow{2}{*}{$\begin{array}{l}\text { Intensityl } \\
\text { Duration } \\
\end{array}$} & \multirow{2}{*}{$\begin{array}{l}\text { Control } \\
\text { group }\end{array}$} & \multirow[t]{2}{*}{ Outcome measures } & \multirow[t]{2}{*}{ Outcomes } \\
\hline & & & & & Modality & \begin{tabular}{|l|} 
Distance \\
or contact \\
healing
\end{tabular} & Blinding procedure(s) & & & & & \\
\hline \multirow[t]{2}{*}{$\begin{array}{l}\text { Assefi, } \\
\text { Bogart [13] }\end{array}$} & $\begin{array}{l}\text { Factorial } \\
\text { design } \\
\text { (phase III } \\
\text { RCT) }\end{array}$ & $\begin{array}{l}\text { Subjective } \\
\text { pain }\end{array}$ & Fibro-myalgia & $\begin{array}{l}93 \\
\text { Distance Reiki } \\
=24 \\
\text { Sham }=23\end{array}$ & Reiki & Distance & $\begin{array}{l}\text { Hands in sending mode } \\
\text { (undefined) from } 2 \text { feet away } \\
\text { Sham- Actor to mimic } \\
\text { movements with cognitive } \\
\text { tasks to minimise } \\
\text { unconscious healing } \\
\text { intentions }\end{array}$ & No & $\begin{array}{l}\text { I: } 2 \text { mins } \times 12 \\
\text { standard hand } \\
\text { positions ( } 30 \\
\text { mins) } \\
\text { D: twice } \\
\text { weekly x } 8 \\
\text { weeks }\end{array}$ & Sham & $\begin{array}{l}\text { VAS pain scale at weeks } \\
4,8 \text { and } 20 .\end{array}$ & No improvement in pain \\
\hline & & & & $\begin{array}{l}\text { Reiki }=23 \\
\text { Sham }=23\end{array}$ & & Contact & $\begin{array}{l}\text { Actor to mimic hand } \\
\text { movements }\end{array}$ & & & Sham & & \\
\hline $\begin{array}{l}\text { Walach, } \\
\text { Boesch [42] } \\
\text { UK }\end{array}$ & $\begin{array}{l}2 \times 2 \\
\text { factorial } \\
\text { design } \\
\text { (Phase III } \\
\text { RCT) }\end{array}$ & $\begin{array}{l}\text { Mental } \\
\text { Health } \\
\text { Scores }\end{array}$ & $\begin{array}{l}\text { Chronic } \\
\text { fatigue or } \\
\text { severe } \\
\text { idiopathic } \\
\text { chronic } \\
\text { fatigue }\end{array}$ & $\begin{array}{l}409 \\
\text { Distance }=102 \\
\text { Blind distance } \\
\text { healing = 105 } \\
\text { Waitlist =108 } \\
\text { Blind waitlist = } \\
94\end{array}$ & $\begin{array}{l}\text { Spiritual } \\
\text { healing }\end{array}$ & Distance & $\begin{array}{l}\text { No contact between healer } \\
\text { and participant (minimum } \\
100 \mathrm{~km} \text { apart) }\end{array}$ & Yes & $\begin{array}{l}\text { I: as per } \\
\text { healer } \\
\text { (mean } 20 \\
\text { minutes) } \\
\text { D: } 6 \text { months }\end{array}$ & \begin{tabular}{|l|} 
Waitlist \\
(with or \\
without \\
knowledge \\
of \\
allocation)
\end{tabular} & $\begin{array}{l}\text { SF-36 MHCS pre and } \\
\text { post study. }\end{array}$ & $\begin{array}{l}\text { No improvement in } \\
\text { mental health }\end{array}$ \\
\hline $\begin{array}{l}\text { Tsang, } \\
\text { Carlson [43] } \\
\text { Canada }\end{array}$ & $\begin{array}{l}\text { Counter- } \\
\text { balanced } \\
\text { crossover } \\
\text { pilot study }\end{array}$ & fatigue & $\begin{array}{l}\text { Cancer } \\
\text { outpatients }\end{array}$ & $\begin{array}{l}16 \\
\text { Reiki }=8 \\
\text { Rest }=8\end{array}$ & Reiki & & & No & $\begin{array}{l}\text { I: Healer } \\
\text { determined, } \\
\text { average } 45 \\
\text { mins } \\
\text { D: daily x } 5 \\
\text { days; } 1 \text { week } \\
\text { rest; } 2 \\
\text { sessions; } 2 \\
\text { weeks no } \\
\text { treatment }\end{array}$ & $\begin{array}{l}\text { Resting } \\
\text { crossover } \\
\text { group }\end{array}$ & $\begin{array}{l}\text { FACT }^{*} \text { - fatigue subscale, } \\
\text { FACT-general version, } \\
\text { ESAS }^{*} \text { pre and post } \\
\text { sessions. }\end{array}$ & $\begin{array}{l}\text { Significant reduction in } \\
\text { fatigue levels }(p<0.01) \text {, } \\
\text { improved } Q O L \\
(p<0.05) ; \\
\text { No improvement in pain } \\
\text { or anxiety. }\end{array}$ \\
\hline $\begin{array}{l}\text { Tsubono, } \\
\text { Thomlinson } \\
\text { [44] } \\
\text { US }\end{array}$ & $\begin{array}{l}\text { Pilot } \\
\text { study }\end{array}$ & Pain levels & $\begin{array}{l}\text { Chronic pain } \\
\text { with no clear } \\
\text { organic } \\
\text { disease }\end{array}$ & $\begin{array}{l}17 \\
\text { Healing }=7 \\
\text { Control }=9\end{array}$ & $\begin{array}{l}\text { Spiritual } \\
\text { healing. } \\
\text { All } \\
\text { participants } \\
\text { meditated } \\
\text { daily }\end{array}$ & Distance & $\begin{array}{l}\text { Separated by country. } \\
\text { Participants attended } 1 \text { hour } \\
\text { meditation session with } \\
\text { healer. Healer returned } \\
\text { overseas. Participants did not } \\
\text { know if they were recipients } \\
\text { of distance healing. }\end{array}$ & No & $\begin{array}{l}\text { I: } 20 \text { mins } \\
\text { (meditation); } \\
\text { healing as } \\
\text { per healer } \\
\text { D: daily x } 2 \\
\text { months }\end{array}$ & \begin{tabular}{|l|} 
Daily \\
meditation
\end{tabular} & $\begin{array}{l}\text { VAS pain scale, MPQ } \\
\text { (PPI and PRI) twice pre } \\
\text { and post intervention }\end{array}$ & $\begin{array}{l}\text { reduced VAS pain } \\
\text { scale scores } \\
(p=0.0056) ; \\
\text { reduced PPI scores } \\
(p=0.016) ;\end{array}$ \\
\hline
\end{tabular}




\begin{tabular}{|c|c|c|c|c|c|c|c|c|c|c|c|c|}
\hline \multirow{2}{*}{$\begin{array}{l}\text { Authorl } \\
\text { country }\end{array}$} & \multirow{2}{*}{\begin{tabular}{|l|} 
Study \\
design
\end{tabular}} & \multirow{2}{*}{\begin{tabular}{|l|} 
Primary \\
outcomes \\
\end{tabular}} & \multirow[t]{2}{*}{ Population } & \multirow[t]{2}{*}{ Number } & \multicolumn{3}{|c|}{ Intervention content } & \multirow{2}{*}{\begin{tabular}{|l|}
$\begin{array}{l}\text { Expectancy } \\
\text { measures }\end{array}$ \\
\end{tabular}} & \multirow{2}{*}{\begin{tabular}{|l|} 
Intensityl \\
Duration \\
\end{tabular}} & \multirow{2}{*}{\begin{tabular}{|l|} 
Control \\
group \\
\end{tabular}} & \multirow[t]{2}{*}{ Outcome measures } & \multirow[t]{2}{*}{ Outcomes } \\
\hline & & & & & Modality & \begin{tabular}{|l|} 
Distance \\
or contact \\
healing \\
\end{tabular} & Blinding procedure(s) & & & & & \\
\hline \multirow[t]{2}{*}{$\begin{array}{l}\text { Richeson, } \\
\text { Spross [45] }\end{array}$} & $\begin{array}{l}\text { Pilot } \\
\text { Study }\end{array}$ & $\begin{array}{l}\text { Anxiety, } \\
\text { Depression } \\
\text { Pain }\end{array}$ & $\begin{array}{l}\text { Older adults } \\
\text { living in the } \\
\text { community }\end{array}$ & $\begin{array}{l}25 \\
\text { Reiki = } 13 \\
\text { Control = } 12\end{array}$ & Reiki & Contact & No & No & $\begin{array}{l}\text { I: } 45 \text { mins } \\
\text { D: weekly } x \\
8 \text { weeks }\end{array}$ & Waitlist & $\begin{array}{l}\text { HAS, Geriatric } \\
\text { Depression Scale (Short } \\
\text { Form), Faces Pain } \\
\text { Scale, HR, BP pre and } \\
\text { post intervention. }\end{array}$ & $\begin{array}{l}\text { Improved anxiety, } \\
\text { pain, and depression } \\
\text { (all } p<0.001 \text { ) } \\
\text { No change in } \mathrm{HR}, \mathrm{BP}\end{array}$ \\
\hline & & & & Reiki $=13$ & & contact & No & & & & & \\
\hline $\begin{array}{l}\text { Easter and } \\
\text { Watt [46] } \\
\text { Scotland }\end{array}$ & $\begin{array}{l}\text { Pilot } \\
\text { study }\end{array}$ & $\begin{array}{l}\text { Pain, well } \\
\text { being }\end{array}$ & Arthritis & $\begin{array}{l}60 \\
\text { Blind } \\
\text { Healing=18 } \\
\text { Healing =15 } \\
\text { Blind control } \\
=15 \\
\text { Control =12 }\end{array}$ & $\begin{array}{l}\text { Distance } \\
\text { healing }\end{array}$ & Distance & $\begin{array}{l}\text { No contact between } \\
\text { healers and participants. }\end{array}$ & Yes & $\begin{array}{l}\text { I: } 15 \text { mins } \\
\text { daily to } 20 \text { - } \\
40 \text { mins } \\
\text { weekly } \\
\text { D: } 6 \text { weeks }\end{array}$ & $\begin{array}{l}\text { Waitlist. } \\
\text { Usual } \\
\text { medical } \\
\text { treatment }\end{array}$ & $\begin{array}{l}\mathrm{MPQ}^{*}, \mathrm{GHQ}-12 \text { at } \\
\text { baseline, post } \\
\text { intervention ( } 6 \text { weeks) } \\
\text { and } 1 \text { month follow up }\end{array}$ & $\begin{array}{l}\text { No improvement in } \\
\text { pain or general health } \\
\text { in blinded groups or } \\
\text { expectancy groups. }\end{array}$ \\
\hline $\begin{array}{l}\text { Catlin and } \\
\text { Taylor-Ford } \\
\text { [47] } \\
\text { US }\end{array}$ & $\begin{array}{l}\text { Phase II } \\
\text { RCT }\end{array}$ & $\begin{array}{l}\text { Comfort, } \\
\text { well-being }\end{array}$ & $\begin{array}{l}\text { Cancer } \\
\text { patients } \\
\text { receiving } \\
\text { outpatient } \\
\text { chemo- } \\
\text { therapy }\end{array}$ & $\begin{array}{l}189 \\
\text { Reiki }=63 \\
\text { Sham }=63 \\
\text { Control = } 63\end{array}$ & Reiki & Contact & $\begin{array}{l}\text { Sham - Actor mimicking } \\
\text { hand movements in specific } \\
\text { order as per Reiki therapist. } \\
\text { Actor does not believe in } \\
\text { energy transfer, did mental } \\
\text { arithmetic during session. } \\
\text { Infusion centre nurse and } \\
\text { patients blinded to whether } \\
\text { Sham Reiki or Reiki was } \\
\text { being offered that day. }\end{array}$ & No & $\begin{array}{l}\text { I: } 20 \text { mins } \\
\text { D: one } \\
\text { session }\end{array}$ & $\begin{array}{l}2.5 \mathrm{hrs} \\
\text { intra- } \\
\text { venous } \\
\text { chemo- } \\
\text { therapy } \\
\text { seated in } \\
\text { a lounge } \\
\text { chair }\end{array}$ & $\begin{array}{l}\text { Healing Touch Comfort } \\
\text { Questionnaire; Well- } \\
\text { Being Analog Scale pre } \\
\text { and post intervention }\end{array}$ & $\begin{array}{l}\text { No difference between } \\
\text { Reiki and sham Reiki } \\
\text { groups }\end{array}$ \\
\hline $\begin{array}{l}\text { Clark, } \\
\text { Cortese- } \\
\text { Jlmenez } \\
{[48]} \\
\text { US }\end{array}$ & $\begin{array}{l}\text { Pre and } \\
\text { post-test }\end{array}$ & $\begin{array}{l}\text { Psychologic } \\
\text { al distress, } \\
\text { QOL }\end{array}$ & $\begin{array}{l}\text { Chemotherapy } \\
\text { induced } \\
\text { peripheral } \\
\text { neuropathy }\end{array}$ & $\begin{array}{l}36 \\
\text { Reiki }=9 \\
\text { Yoga }=9 \\
\text { Meditatior } \\
\text { Education }\end{array}$ & Reiki & Contact & No & No & $\begin{array}{l}\text { I: } 1 \text { hour } \\
\text { D: weekly x } \\
6 \text { weeks }\end{array}$ & $\begin{array}{l}\text { Holistic } \\
\text { education } \\
\text { class on } \\
\text { physical, } \\
\text { psychologic } \\
\text { al, social } \\
\text { aspects of } \\
\text { neuropathy }\end{array}$ & $\begin{array}{l}\text { BSI }{ }^{*}, \text { FACT- Gynaecology } \\
\text { Oncology Neurotoxicity } \\
\text { Scale, Mindful Attention } \\
\text { Awareness Scale pre and } \\
\text { post intervention }\end{array}$ & $\begin{array}{l}\text { No improvement in } \\
\text { psychosocial distress, } \\
\text { neurotoxicity }\end{array}$ \\
\hline Lu, Hart [49] & $\begin{array}{l}\text { Repeated } \\
\text { measures }\end{array}$ & $\begin{array}{l}\text { Pain level, } \\
\text { joint }\end{array}$ & osteoarthritis & $\begin{array}{l}19 \\
\text { Healing }=12\end{array}$ & $\begin{array}{l}\text { Healing } \\
\text { Touch }\end{array}$ & Contact & No & No & \begin{tabular}{|l|} 
I: 20 mins \\
D: $3 x$ week/ 6
\end{tabular} & $\begin{array}{l}\text { Friendly } \\
\text { visit - }\end{array}$ & $\begin{array}{l}\text { lowa Pain Thermometer } \\
\text { pre and post sessions, }\end{array}$ & $\begin{array}{l}\text { Decreased pain severity } \\
(p=0.02) \text { and pain }\end{array}$ \\
\hline
\end{tabular}




\begin{tabular}{|c|c|c|c|c|c|c|c|c|c|c|c|c|}
\hline \multirow{2}{*}{$\begin{array}{l}\text { Authorl } \\
\text { country }\end{array}$} & \multirow{2}{*}{$\begin{array}{l}\text { Study } \\
\text { design }\end{array}$} & \multirow{2}{*}{\begin{tabular}{|l|} 
Primary \\
outcomes
\end{tabular}} & \multirow[t]{2}{*}{ Population } & \multirow[t]{2}{*}{ Number } & \multicolumn{3}{|c|}{ Intervention content } & \multirow{2}{*}{\begin{tabular}{|l|}
$\begin{array}{l}\text { Expectancy } \\
\text { measures }\end{array}$ \\
\end{tabular}} & \multirow{2}{*}{\begin{tabular}{|l|} 
Intensityl \\
Duration
\end{tabular}} & \multirow{2}{*}{$\begin{array}{l}\text { Control } \\
\text { group }\end{array}$} & \multirow[t]{2}{*}{ Outcome measures } & \multirow[t]{2}{*}{ Outcomes } \\
\hline & & & & & Modality & \begin{tabular}{|l|} 
Distance \\
or contact \\
healing \\
\end{tabular} & Blinding procedure(s) & & & & & \\
\hline US & design & $\begin{array}{l}\text { function, } \\
\text { mobility, } \\
\text { depression }\end{array}$ & & Control $=7$ & & & & & & $\begin{array}{l}\text { weekly } \\
\text { talks on } \\
\text { selected } \\
\text { topics }\end{array}$ & $\begin{array}{l}\text { BPI-SF* }{ }^{*} \text { WOMAC, } \\
\text { goniometer, PHQ-9* at } \\
\text { baseline, post } \\
\text { intervention, } 3 \text { week } \\
\text { follow up }\end{array}$ & $\begin{array}{l}\text { interference }(p=0.02) \text { at } 6 \\
\text { and } 9 \text { weeks; } \\
\text { Improved joint function } \\
\text { (extensor lag and } \\
\text { extension better knee) } \\
(p=0.03) \text { at } 6 \text { weeks; no } \\
\text { improvement in } \\
\text { depression. }\end{array}$ \\
\hline $\begin{array}{l}\text { Pedersen, } \\
\text { Johannesse } \\
\mathrm{n}[50] \\
\text { Denmark }\end{array}$ & $\begin{array}{l}\text { Secondary } \\
\text { analysis of } \\
\text { phase III } \\
\text { RCT }\end{array}$ & $\begin{array}{l}\text { QOL, } \\
\text { depressive } \\
\text { symptoms, } \\
\text { mood, sleep } \\
\text { quality }\end{array}$ & $\begin{array}{l}\text { Completed } \\
\text { colorectal } \\
\text { cancer } \\
\text { treatment }\end{array}$ & $\begin{array}{l}783 \\
\text { Randomised } \\
\text { healing = 58 } \\
\text { Control }=55 \\
\text { Self-select } \\
\text { healing }=82 \\
\text { Self-select } \\
\text { control = } 52\end{array}$ & $\begin{array}{l}\text { Energy } \\
\text { healing }\end{array}$ & Contact & No & No & $\begin{array}{l}\text { I: not stated } \\
\text { D: } 4 \text { sessions } \\
\text { over } 2 \\
\text { months }\end{array}$ & \begin{tabular}{|l|} 
Offered \\
one free \\
session \\
after study \\
completion
\end{tabular} & $\begin{array}{l}\text { FACIT-sp* (Danish); BDI*- } \\
\text { II; POMS (Danish); } \\
\text { Pittsburgh Sleep Quality } \\
\text { Index at baseline, post 2nd, } \\
3^{\text {rd }} \text { healing, } 6 \text { weeks (CG), } \\
8 \text { and } 16 \text { weeks. }\end{array}$ & $\begin{array}{l}\text { No overall } \\
\text { improvement in QOL, } \\
\text { depression, mood } \\
\text { sleep quality. }\end{array}$ \\
\hline $\begin{array}{l}\text { FitzHenry, } \\
\text { Wells [51] } \\
\text { US }\end{array}$ & $\begin{array}{l}\text { Pilot } \\
\text { study }\end{array}$ & $\begin{array}{l}\text { Fatigue, } \\
\text { QOL, } \\
\text { anxiety/ } \\
\text { depression }\end{array}$ & $\begin{array}{l}\text { Outpatient } \\
\text { radiotherapy } \\
\text { cancer } \\
\text { patients }\end{array}$ & $\begin{array}{l}41 \\
\text { Healing }=21 \\
\text { Sham }=20\end{array}$ & $\begin{array}{l}\text { Healing } \\
\text { Touch }\end{array}$ & $\begin{array}{l}\text { Contact } \\
\text { (no } \\
\text { touch) }\end{array}$ & $\begin{array}{l}\text { Participants had a drape at } \\
\text { neck or blindfold to ensure } \\
\text { blinding to treatment } \\
\text { delivery }\end{array}$ & no & $\begin{array}{l}\text { I: } 45 \text { mins } \\
\text { D: weekly x } \\
7 \text { weeks }\end{array}$ & Sham & $\begin{array}{l}\text { HAD Scale, Brief Fatigue } \\
\text { Inventory, FACT-B } \\
\text { (Breast) pre and post } \\
\text { intervention }\end{array}$ & $\begin{array}{l}\text { No improvement in } \\
\text { fatigue, QOL }\end{array}$ \\
\hline
\end{tabular}

Key: Minutes (mins); hours (hrs); Control Group (CG); Number of participants (N) or (n); Primary Endpoint (PE); McGill Pain Questionnaire (MPQ); Short Form 36 (SF-36); Profile of Mood States (POMS); Visual Analog Scale (VAS); Mental Health Component Summary (MHCS), Asthma Quality of Life Questionnaire (AQLQ); Ritchie Articular Index (RAl); Early Morning Stiffness (EMS); Stanford Health Assessment Questionnaire (SHAQ); Eysenck Personality Questionnaire (EPQ); McGill Pain Questionnaire (MPQ); Beck Depression Inventory (BDI); Cognitive Brain Therapy (CBT); Functional Assessment of Chronic IIIness Therapy-Spiritual Well-Being Scale (FACIT-sp); Hamilton Anxiety Scale (HAS); Brief Pain Interview Short Form (BPI-SF); Western Ontario and McMasters University Osteoarthritis Index (WOMAC); Patient Health Questionnaire -9 (PHQ-9); Power as Knowing Participation in Change Tool (PKPCT); Pain Disability Index (PDI); Chronic Pain Self Efficacy Scale (CPSS); Functional Assessment of Cancer Therapy (FACT);

Edmonton Symptom Assessment Scale (ESAS); Brief Symptom Inventory (BSI); Perceived Stress Scale (PSS); Cognitive Brain Therapy (CBT); Present Pain Index (PPI); Pain Rating Index (PRI) 


\section{Control and/or comparison groups}

Ten energy healing studies included a usual care control group, defined as subjects who received usual medical treatment for example continuation of antihypertensive treatment or low salt diet [27], no contact with investigators [31], four doctor (and blind nurse assessor) visits [33], no active pain clinic therapy [29], standard cancer treatment [37], receipt of intravenous chemotherapy medications for between two and five hours [47], reading material of their choice at the investigator's office [12], nursing home care including assistance with hygiene, medication, meals, vital signs and monitoring of activity [32], or was not clearly defined in two studies [41, 50]. A usual care [37] or resting [43] crossover control group or a waitlist control group [30, 35, 45] was used in four studies allowing all participants to eventually receive the intervention. The remaining studies used a variety of control and/or comparison groups including expectancy $[39,46,52]$; a sham healing group using an actor to mimic movements of the healer $(n=11)[12,13,28,31,32,34,38,40$, $41,47,51$ ], relaxation [36], progressive muscle relaxation and deep breathing [12]; massage therapy [37], yoga [48], meditation $(n=2)[48,53]$, presence $(n=2)[37,54]$, friendly visit [49], education [48], distance healing $(n=2)[13,39]$ or multiple distance healing comparison groups (including contact, no contact and a group that wore an amulet given healing) [35]. A further study compared a selfselected healing, self-selected control group and randomised control group to a randomised healing intervention [50].

\section{Expectancy and belief measures}

Seven energy healing studies adequately assessed for belief or expectancy effects $[12,34,35,38,39$, $42,46]$. In two energy healing studies, participants were divided into two groups whereby one group were informed they would receive energy healing at a distance whilst the other group remained blinded to treatment condition $[42,46]$. Treatment outcomes were then compared among participants allocated to distant healing groups who were expecting to receive healing compared to those who remained blinded. In a second study participants were first randomly allocated to distance healing or proximal healing groups. In the proximal healing group, participants were then 
randomly assigned to either healing or sham healing whilst those in the distance healing group were assigned to either receive distance healing or to receive no healing [34]. A third distance healing study randomly allocated participants to treatment or waitlist control groups. Those allocated to the treatment group were quasi-randomly allocated to either distance healing with healer contact via telephone prior to the commencement of the intervention only; no healer contact but were sent an amulet to wear that was purported to transmit healing energy; or were treated anonymously by more than one healer [35]. Alternatively, expectation was assessed by deception, whereby participants allocated to a 'mimic' Reiki group or distant healing control group were informed they would receive distant healing, however did not [39]. A revised Belief in Personal Control Questionnaire was used to assess for belief in a higher power in one study [12], or belief in group assignment was asked at baseline and post intervention [38]. Expectation of receiving distance healing was significantly associated with positive outcomes for adults with a diagnosed chronic illness or chronic fatigue syndrome [52]; and was partially supported (by small to moderate clinically significant effect sizes) in one study for adults with arthritis [46]. The Goldman Shore [39] RCT found that distance healing was not significantly associated with positive outcomes among adults with depression or other chronic illnesses. Authors conclude that belief in the assistance of a higher power was significantly associated with improvements in pain among women in one pilot study, however elements of the pain rating intensity subscale were improved rather than the total PRI score. [12]. Belief in the effectiveness of healing was not significantly associated with positive outcomes for adults with chronic pain or arthritis $[34,46]$. Health related quality of life was not significantly associated with belief in group allocation in one study [38].

\section{Assessment of risk of bias}

Three energy healing studies had a low risk of bias $[13,52,53]$, whilst an unclear risk of bias was found in two studies due to unclear randomisation procedures [39] and allocation procedures [39, 46]. The remaining studies contained a high risk of bias sufficient to weaken the plausibility of results due to an inability to blind the energy healing practitioner ( $n=22)$; no allocation concealment 
procedures $(n=2)$; lack of blinding of outcome assessors for patient reported outcomes $(n=3)$; incomplete outcome data for short term outcomes (2-6 weeks) $(n=3)$; or long term outcomes (>6 weeks) ( $n=2)$; or selective reporting $(n=1)$ [26]. There was less chance for bias to occur with distant healing as there is no physical contact between the healer and the participants. Detailed information of the assessment of risk of bias is provided in a supplementary appendix (Appendix 1).

\section{Summary of energy healing interventions}

Half of the included energy healing studies ( $n=13$ studies) found significantly positive outcome for health and/or wellbeing. Populations of studies that demonstrated significant results included adults with cancer (4 studies); chronic pain (7 studies) chronic illnesses (3 studies), and arthritis (3 studies). Significant results were demonstrated in all energy healing modalities including spiritual healing, Reiki, Therapeutic Touch and Johnston's Healing (similar to Therapeutic Touch, but without touch) Significant outcomes of included phase III studies were mood disturbance, fatigue and quality of life $[35,37]$. Primary and secondary outcomes of included phase II RCT's and pilot studies that were significant included pain ( $\mathrm{n}=8$ studies), quality of life, coping and health locus of control, anxiety, selfesteem, psychological distress, fatigue, joint function, depression. Five studies identified adverse effects in a minority of recipients including increased anxiety; excess energy worsening sleep or depressed mood; transient headache or crying $(n=2)$; temporary severe pain and fever (fibromyalgia patients; $n=4$ ) (that was followed by rapid improvement in symptoms) associated with healing [13, $27,28,45,53]$.

\section{Key elements of energy healing interventions}

Utilising the same healer(s) for all sessions was a key element of 12 out of 13 studies that had significant results $[12,28,29,31,32,35,37,39,43,45,49,53]$. A further seven studies maintained the same healer(s) for all sessions however results of these studies were not significant $[13,27,33$, $40,46,47,50,55]$. 
Eleven out of 13 energy healing interventions with significant results involved large groups with greater than five participants allocated per healer $[12,29,31,32,35,37,39,43,45,49,53,56]$. Laying of hands either on or a few inches above the body was utilised in 22 out of the 26 included studies, of which twelve studies generated significant results $[12,28,29,31,32,37-39,43,45,48$, 49]. A meditative component was included in five studies with significant results $[28,32,37,38,53]$. Healers that were not restricted in their practice were utilised in 20 studies of which 9 generated significant results $[29,31,32,35,37,39,43,49,53,56]$. Directed healing towards a specific health outcome or well-being was used in 18 studies of which nine demonstrated a significant result [28, 31, $32,37-39,45,48,49]$.

Verbal communication prior to energy healing at a distance $[35,53]$ or contact energy healing $[28$, $29,32,37,48]$ took place between healer and recipient in six studies with significant outcomes, however, verbal communication was not used during the intervention period in seven studies with significant results $[12,31,38,39,43,45,49,56]$. A specific visualisation component was not included in two studies that had significant results $[40,52]$. This may reflect that adequate information about healing interventions was not provided, or that visualisation was not a necessary component of effective healing interventions

The information provided to the healers in the majority of studies $(n=20)$ was not clearly defined, of which eleven studies were significant $[12,28,29,31,32,37-39,43,45,49]$.

\section{Discussion}

Statistically significant improvements were demonstrated in half of the energy healing studies for 13 different outcomes. Three significant outcomes were identified within phase III studies for 1) mood disturbance, 2) fatigue, and 3) quality of life. Twelve significant outcomes were identified in phase II and pilot studies for 1) pain, 2) quality of life, 3) hopelessness related to coping, 4) health locus of control, 5) anxiety, 6) self-esteem, 7) psychological distress, 8) fatigue, 9) joint function, 10) 
depression, 11) vitality, and 12) physical function, however overall findings remain inconclusive. These results reflect the preliminary nature of energy healing research, which is also mirrored by inconclusive evidence from a previous Cochrane review for depression and/or anxiety symptoms [25]. Evidence supporting the routine use of energy healing to manage the symptom burden associated with NCDs is questionable, due to the quality of the trials that were included. Future trials need to minimise the risk of bias and adhere to the CONSORT statement. Strengthening the design of future energy healing trials and developing an energy healing taxonomy would do much to help address the inconclusive evidence that currently exists about the efficacy of energy healing as a selfmanagement strategy. Key elements of successful interventions identified and recommended for future interventions include: maintenance of healers and their usual unrestricted practices for the duration of an intervention, incorporation of a meditative component, and limited verbal communication prior to healing delivery.

Healing was significantly associated with improvements in osteoarthritic, chronic pain or cancer pain in eight studies but not for the arthritic, chronic pain or diabetic neuropathic pain in a further eight studies, which may suggest that healing effects are highly individual and specific to certain contexts. Ambiguous results could also be due to sampling error given the small number of participants included in these studies. It has been suggested that RCTs alone may not be the ideal design for assessing healing effects [50]. Blinding procedures may dilute healing effects by creating uncertainty between patients and healers, nor does it produce ecological validity that is consistent with real life practice interactions between healers and their subjects $[34,42,50]$. Further, the use of disease specific outcomes does not capture the broader impact of healing and is antithetical to the approach of healers who focus on the whole person [4]. In most instances physical symptoms that healees rated as important were not completely alleviated by healing, however improvements were demonstrated in general health and well-being, enhanced coping ability and decreased symptom burden without explicitly changing the nature of the symptoms [4]. Further, effects of healing such as crying may be reported as an adverse outcome, however this may not be the case if it is 
associated with emotional release [57]. A mixed methods design that incorporates qualitative approaches may be the ideal design to shed light on the ambiguous results from this review, as well as previous reviews of energy healing $[47,58]$.

\section{Relevance of current results to the chronic care literature}

Inconsistencies in healing effects may be attributable to issues of secondary gain among adults with NCDs. Attachment to illness symptomatology is likely to occur on an unconscious level and may be accompanied by repressed negative emotional states such as anger that may compound the experience of pain, decreased health and psychosocial dysfunction [59]. Energy healing offers the potential for a shift in physiological state and present moment awareness that may be a first step in altering the perspectives that sustain symptom burden. Energy healing also has the potential to "facilitate personal growth through a deeper connection to the spiritual aspects of life, thereby reducing stress..." and alleviating illness symptoms [39, p. 43]. It may also improve self-confidence, emotional balance, inner strength, acceptance of health status and relationships with friends and family members [4]. In instances of chronic pain where pain is often disproportionate to tissue damage, and underlying causes are not easily identified, energy healing has the capacity to produce a deep relaxation effect, thereby decreasing muscle tension and shifting the perspective of the patient away from an internal focus on their symptoms towards a more positive self-perception [29, 44].

\section{Limitations}

This review is limited by the inability to conduct meta-analyses given the heterogeneous nature of included studies. The paucity of methodologically strong studies is a major limitation of this review. Individual interventions frequently lacked adequate detail, which limits conclusions drawn around elements of energy healing interventions that may enhance outcomes, and denies study replication. This lack of detail may indicate that the provision of information about the recipients for example is not a necessary component for healing effects to take place. 


\section{Implications for practice}

Patient preference is an important component of all evidence based practice [60]. Energy healing is immensely personal and is a self-management strategy that is unlikely to appeal to everyone. The need for informed consent is a crucial consideration for energy healing research as well as for clinical practice [14]. There is a potential for risk of bias within studies given the high motivation of participants to participate in their own healing process [39]. Having an open minded and/or positive approach (positive expectancy) to healing may precede healing effects, which likely reflects users of healing in the community $[35,42]$. Further research is required to understand characteristics of adults who use energy healing and whether there are unique circumstances or life experiences that influence the choice to engage in healing practices.

It has been previously suggested that given the secular nature of clinical settings delivering care to community based populations, implementation of energy healing interventions will inevitably be competing with other therapies for which there exists more supportive empirical evidence including psychological therapy $[17,61]$. This review identified two phase III energy healing interventions that generated clinically significant improvements in quality of life, with the outcome of one study among adults with chronic illnesses equivalent to improvements previously demonstrated for psychotherapy (mean difference 0.66 SD's) [35]. Further studies are required in order to replicate these effects to provide further evidence supporting their efficacy [17]. Clinical decision making regarding the applicability of energy healing to practice should also be guided by a framework that considers baseline risk of poor outcomes, responsiveness to energy healing as a treatment option, and participant vulnerability to adverse effects [62].

\section{Implications for research}

Preliminary healing studies demonstrate feasibility of conducting larger adequately powered, randomised controlled trials, although many studies did not progress to this third stage. In order to determine the true impact of healing on NCD symptoms in the community, further large scale 
controlled trials, and ideally studies incorporating mixed methods, are required to ascertain the 'uniqueness of perceived healing effects' among this population [24]. To advance research on energy healing controlled trials should include sample size calculations that are powered to the primary outcome, clearly define primary and secondary outcome measures, avoid the use of multiple outcome measures (fishing), avoid disease specific measures, focus on one particular NCD at a time or use separate analyses for each NCD, and clearly report intervention designs and healer practices to allow for replication. Optimal group size and the importance of continuity of healer across sessions will also need to be determined with further research. The aim of clinical trials of novel nonpharmacological therapies should progress from "...simple demonstration of statistical superiority..." to include the assessment of differential effects with due consideration of the impact of clinical characteristics and the role of adverse effects that persist over time $[62, p .2]$.

\section{Conclusion}

NCDs have significant cost implications for the health care system. Given potential benefits of energy healing interventions as an adjunct self-management strategy, this review recommends the need for further controlled trials and mixed methods studies that adhere to the CONSORT statement. Future studies are required to replicate positive results and understand the NCD population groups, physiological and psychological outcomes, patient specific factors and elements of energy healing interventions that are favourable towards positive outcomes. Future intervention designs may benefit from the maintenance of healers and their usual unrestricted practices, inclusion of a meditative component, and limiting verbal communication prior to healing. Ethical considerations are important in undertaking energy healing interventions in clinical settings, and due consent and consideration is needed in respect of cultural, religious and individual values and preferences.

\section{Conflict of Interest}

The authors declare there is no conflict of interest. 


\section{References:}

1. Fava GA, Sonino N. Psychosomatic medicine. Int J Clin Pract. 2010;64:1155-61.

2. Schlitz M, Amorok T, Micozzi M. Consciousness and healing: Integral approaches to mindbody medicine. St Louis: Elseveir Churchill; 2005:1-585.

3. Wagner $\mathrm{EH}$, Austin $\mathrm{BT}$, Davis $\mathrm{C}$, et al. Improving chronic illness care: translating evidence into action. Health affairs. 2001;20:64-78.

4. Bishop FL, Barlow F, Walker J, et al. The Development and Validation of an Outcome Measure for Spiritual Healing: A Mixed Methods Study. Psychotherapy and Psychosomatics. 2010;79:350-62.

5. Rao A, Sibbritt D, Phillips JL, et al. Prayer or spiritual healing as adjuncts to conventional care: a cross sectional analysis of prevalence and characteristics of use among women. BMJ Open. 2015;5. 6. Yates J, Mustian K, Morrow G, et al. Prevalence of complementary and alternative medicine use in cancer patients during treatment. Supportive Care in Cancer. 2005;13:806-11.

7. Clarke TC, Blck LI, Stussman BJ, et al. Trends in the use of complementary health approaches among adults: United States 2002-2012. National health statistics report no. 79. Hyattsville, MD: National Institutes of Health, 2015.

8. Brennan BA. Hands of light. A guide to healing the human energy field. New York: Bantam Books; 1988.

9. Robinson J BF, Dolk H. Therapeutic touch for anxiety disorders. The Cochrane Library. 2009.

10. Stefano GB, Fricchione GL, Slingsby BT, et al. The placebo effect and relaxation response: neural processes and their coupling to constitutive nitric oxide. Brain Research Reviews. 2001;35:119.

11. Benor DJ. Spiritual healing: Scientific validation of a healing revolution: Wholistic Healing Pubns; 2001:1-597.

12. Dressen LJ, Singg S. Effects of Reiki on pain and selected affective and personality variables of chronically ill patients. Subtle Energies \& Energy Medicine Journal Archives. 1998;9.

13. Assefi N, Bogart A, Goldbery J, et al. Reiki for the treatment of fibromyalgia: A randomized controlled trial. J Altern Complement Med. 2008;14:1115-22.

14. Jonas W, Crawford C. Science and spiritual healing: a critical review of spiritual healing, 'energy' medicine, and intentionality. Alternative Therapies in Health and Medicine. 2003;9:56-61.

15. Strauss SE. Complementary and Alternative Medicine. 16 ed. Kasper DL, Braunwald E, Hauser S, Longo D, Jameson JL, Fauci AS, editors: McGraw-Hill Publication; 2005:66-70.

16. Cheung $\mathrm{C}$, Wyman J, Halcon L, Use of complementary and alternative therapies in community-dwelling older adults. J Altern Complement Med. 2007;13:997-1006.

17. Candy $B$, Jones $L$, Varagunam $M$, et al. Spiritual and religious interventions for well-being of adults in the terminal phase of disease. Cochrane Database of Systematic Reviews. 2012;5:1-52.

18. Adams J, Sibbritt D, Broom A, et al. A comparison of complementary and alternative medicine users and use across geographical areas: A national survey of 1,427 women. BMC complementary and alternative medicine. 2011;11:85.

19. Thomas K, Coleman P. Use of complementary or alternative medicine in a general population in Great Britain. Results from the National Omnibus survey. Journal of Public Health. 2004;26:152-7.

20. Abbot NC. Healing as a therapy for human disease: a systematic review. The Journal of Alternative and Complementary Medicine. 2000;6:159-69.

21. Astin JAP, Harkness EB, Ernst EMDP. The Efficacy of "Distant Healing": A Systematic Review of Randomized Trials. Annals of Internal Medicine. 2000;132:903-10.

22. Crawford C, Sparber A, Jonas W. A systematic review of the quality of research on hands-on and distance healing: clinical and laboratory studies. Alternative Therapies in Health and Medicine. 2003;9:96-104.

23. Roe CA, Sonnex C, Roxburgh EC. Two Meta-Analyses of Noncontact Healing Studies.

EXPLORE: The Journal of Science and Healing. 2015;11:11-23. 
24. Jain S, Mills PJ. Biofield therapies: helpful or full of hype? A best evidence synthesis. Int J Behav Med. 2010;17:1-16.

25. Joyce J, Herbison GP. Reiki for depression and anxiety. The Cochrane Library. 2015.

26. Higgins J, Altman D, Gøtzsche P, et al. Cochrane Bias Methods Group; Cochrane Statistical Methods Group. 2011 The Cochrane Collaboration's tool for assessing risk of bias in randomised trials. BMJ. 2011;343:d5928.

27. Beutler JJ, Attevelt JT, Schouten SA, et al. Paranormal healing and hypertension. British medical journal (Clinical research ed). 1988;296:1491.

28. Redner R, Briner B, Snellman L. Effects of a bioenergo healing technique on chronic pain. Subtle Energies. 1991;2:43-68.

29. Sundblom DM, Haikonen S, Niemi-Pynttäri J, et al. Effect of spiritual healing on chronic idiopathic pain: a medical and psychological study. The Clinical journal of pain. 1994;10:296-302.

30. Eckes Peck SD. The effectiveness of therapeutic touch for decreasing pain in elders with degenerative arthritis. J Holist Nurs. 1997;15:176-98.

31. Gordon A, Merenstein JH, D'Amico F, et al. The effects of therapeutic touch on patients with osteoarthritis of the knee. The Journal of family practice. 1998.

32. Lin YS, Taylor AG. Effects of Therapeutic Touch in Reducing Pain and Anxiety in an Elderly Population. Integrative Medicine. 1998;1:152-62.

33. le Gallez P, Dimmock S, Bird H. Spiritual healing as adjunct therapy for rheumatoid arthritis. British Journal of Nursing. 2000;9:695-712.

34. Abbot NC, Harkness EF, Stevinson C, et al. Spiritual healing as a therapy for chronic pain: a randomized, clinical trial. Pain. 2001;91:79-89.

35. Wiesendanger $\mathrm{H}$, Werthmüller $\mathrm{L}$, Reuter $\mathrm{K}$, et al. Chronically ill patients treated by spiritual healing improve in quality of life: results of a randomized waiting-list controlled study. Journal Of Alternative And Complementary Medicine (New York, NY). 2001;7:45-51.

36. Woods SD, Arnstein P, Cowen RK, et al. Effects of integrating therapeutic touch into a cognitive behavioral pain treatment program. Journal of Holistic Nursing. 2002;20:367-87.

37. Post-White J, Kinney M, Savik K, et al. Therapeutic massage and healing touch improve symptoms in cancer. Integr Cancer Therapies. 2003;2:332-44.

38. Cook CA, Guerrerio JF, Slater VE. Healing touch and quality of life in women receiving radiation treatment for cancer: a randomized controlled trial. Altern Ther Health Med. 2004;10:34-

41.

39. Goldman Shore A. Long-Term Effects Of Energetic Healing On Symptoms Of Psychological Depression And Self-Perceived Stress. Alternative Therapies in Health and Medicine. 2004;10:42-8.

40. Cleland JA, Price DB, Lee AJ, et al. A pragmatic, three-arm randomised controlled trial of spiritual healing for asthma in primary care. Br J Gen Pract. 2006;56:444-9.

41. Gillespie EA, Gillespie BW, Stevens MJ. Painful Diabetic Neuropathy: Impact of an alternative approach. Diabetes Care. 2007;30:999-1001.

42. Walach $\mathrm{H}$, Boesch $\mathrm{H}$, Lewith $\mathrm{G}$, et al. Effectiveness of Distant Healing for Patients with Chronic Fatigue Syndrome: A Randomised Controlled Partially Blinded Trial (EUHEALS).

Psychotherapy and Psychosomatics. 2008;77:158-66.

43. Tsang KL, Carlson LE, Olson K. Pilot crossover trial of Reiki versus rest for treating cancerrelated fatigue. Integrative Cancer Therapies. 2007;6:25-35.

44. Tsubono K, Thomlinson P, Shealy $\mathrm{CN}$. The effects of distant healing performed by a spiritual healer on chronic pain: a randomized controlled trial. Alternative therapies in health and medicine. 2009;15:30-4.

45. Richeson NE, Spross JA, Lutz K, et al. Effects of Reiki on Anxiety, Depression, Pain, and Physiological Factors in Community-Dwelling Older Adults. Research in Gerontological Nursing. 2010;3:187-99. 
46. Easter A, Watt C. It's good to know: How treatment knowledge and belief affect the outcome of distant healing intentionality for arthritis sufferers. Journal of psychosomatic research. 2011;71:86-9.

47. Catlin A, Taylor-Ford RL, editors. Investigation of standard care versus sham Reiki placebo versus actual Reiki therapy to enhance comfort and well-being in a chemotherapy infusion center. Oncology nursing forum; 2011: Onc Nurs Society.

48. Clark PG, Cortese-JImenez G, Cohen E. Effects of Reiki, Yoga or Meditation on the Physical and Psychological Symptoms of Chemotherapy-Induced Peripheral Neuropathy: A Randomised Pilot Study. Journal of Evidence-Based Complementary \& Alternative Medicine. 2012;17:161-71.

49. Lu DF, Hart LK, Lutgendorf SK, et al. The effect of healing touch on the pain and mobility of persons with osteoarthritis: a feasibility study. Geriatr Nurs. 2013;34:314-22.

50. Pedersen CG, Johannessen $\mathrm{H}, \mathrm{Hjelmborg} \mathrm{JV}$, et al. Effectiveness of energy healing on Quality of Life: a pragmatic intervention trial in colorectal cancer patients. Complementary therapies in medicine. 2014;22:463-72.

51. FitzHenry F, Wells $\mathrm{N}$, Slater $\mathrm{V}$, et al. A randomized placebo-controlled pilot study of the impact of healing touch on fatigue in breast cancer patients undergoing radiation therapy. Integr Cancer Ther. 2014;13:105-13.

52. Walach $\mathrm{H}, \mathrm{Bösch} \mathrm{H}$, Lewith $\mathrm{G}$, et al. Effectiveness of distant healing for patients with chronic fatigue syndrome: a randomised controlled partially blinded trial (EUHEALS). Psychotherapy and psychosomatics. 2008;77:158-66.

53. Tsubono K, Thomlinson P, Shealy N. The effects of distant healing performed by a spiritual healer on chronic pain: A randomized controlled trial. Alternative Therapies in Health and Medicine. 2009;15:30-4.

54. Decker S, Wardell DW, Cron SG. Using a Healing Touch Intervention in Older Adults With Persistent Pain: A Feasibility Study. Journal of Holistic Nursing. 2012;30:205-13.

55. Denison B. Touch the pain away: new research on therapeutic touch and persons with fibromyalgia syndrome. Holist Nurs Pract. 2004;18:142-51.

56. Miller RN. Study on the effectiveness of remote mental healing. Medical Hypotheses. 1982;8:481-90.

57. Ryde K, Friedrichsen M, Strang P. Crying: A force to balance emotional among cancer patients in palliative home care. Palliative and Supportive Care. 2007;5:51-9.

58. Miles P, True G. Reiki--review of a biofield therapy history, theory, practice, and research. Alternative Therapies In Health And Medicine. 2003;9:62-72.

59. Worzer W, Kishino N, Gatchel R. Primary, Secondary, and Tertiary Losses in Chronic Pain Patients. Psychological Injury and Law. 2009;2:215-24.

60. Sackett DL, Rosenberg WMC, Gray JAM, et al. Evidence based medicine: what it is and what it isn't1996 1996-01-13 08:00:00.

61. Hodge DR. Using Prayer and Other Forms of Positive Mental Energy in Direct Practice: An Evidence-Based Perspective. Smith College Studies in Social Work. 2010;80:121-38.

62. Fava GA, Guidi J, Rafanelli C, et al. The Clinical Inadequacy of Evidence-Based Medicine and the Need for a Conceptual Framework Based on Clinical Judgment. Psychotherapy and Psychosomatics. 2015;84:1-3. 